\title{
Velocidad de procesamiento en la comprensión morfológica de verbos en niños preescolares con trastorno específico del lenguaje y su relación con el control inhibitorio ${ }^{1}$
}

\author{
Mabel Urrutia ${ }^{2}$, Miriam Roa Inostroza ${ }^{3}$
}

Recibido: 4 de marzo de 2019 / Primera Revisión 17 de agosto de 2019 / Aceptado 20 de enero de 2020.

\begin{abstract}
Resumen. En el último tiempo se ha estudiado la relación del trastorno específico de lenguaje con algunas funciones cognitivas como memoria, atención y funcionamiento ejecutivo, pero pocos estudios en español han investigado la relación de variables psicológicas y lingüísticas en su conjunto. El objetivo de este estudio fue observar la relación entre aspectos cognitivos y lingüísticos, abordando los problemas gramaticales del trastorno específico de lenguaje, específicamente la comprensión morfológica de verbos y el tiempo de reacción en el procesamiento de tales tareas. Los participantes fueron niños y niñas hispanohablantes monolingües con diagnóstico de TEL en el periodo preescolar y un grupo control. A través de un diseño experimental se midió el tiempo de respuesta y la cantidad de aciertos go/no go en el procesamiento de aspectos morfológicos verbales. Tales medidas se relacionaron con el uso de algunas covariables como la inhibición conductual en una tarea no verbal, la velocidad de denominación a nivel de producción lingüística y la memoria de trabajo visual, a partir de una evaluación neuropsicológica. Los resultados mostraron que los niños con TEL se demoraron más tiempo en la identificación de verbos irregulares en comparación con los verbos regulares y un mayor tiempo en la identificación de verbos en tiempo pretérito perfecto simple que verbos en tiempo presente, en comparación con el grupo control. En cuanto al control inhibitorio, los niños con TEL obtuvieron una mayor tasa de errores en tareas de inhibición en comparación con el grupo control y mayor tasa de errores en la comprensión de ambos tipos de verbos y ambos tiempos verbales.
\end{abstract}

Palabras claves: Control Inhibitorio; Funciones Ejecutivas; Trastorno Específico de Lenguaje; Velocidad de procesamiento.

\section{[en] Processing speed of morphological understanding of verbs in preschool children with specific language disorders and its relationship with the inhibitory control}

\begin{abstract}
In recent times the relationship of Specific Language Disorder with some cognitive functions such as memory, attention and executive functioning has been studied, but few studies in Spanish have investigated the relationship of psychological and linguistic variables as a whole The objective of this study was to observe the relationship between cognitive and linguistic aspects, addressing the grammatical problems of Specific Language Disorder, specifically the morphological comprehension of verbs and the reaction time in the processing of such tasks. The participants were monolingual Spanishspeaking boys and girls with a diagnosis of SLI in the preschool period and a control group. Through an experimental design, the response time and the number of hits go / no go in the processing of verbal morphological aspects were measured. Such measures were related to the use other variables such us behavioral inhibition in a non-verbal task, speed of naming at the level of linguistic production and visual working memory from a neuropsychological evaluation. The results showed that children with SLI spent more time in identifying irregular verbs compared to regular verbs and a longer time in the identification of verbs in simple past tense compared to the control group. In terms of inhibitory control, children with SLI had a higher rate of errors in tasks of inhibition compared to the control group and a higher rate of errors in the comprehension of both types of verbs and both verbal tenses.
\end{abstract}

Keywords: Executive Functions; Inhibitory Control; Processing Speed; Specific Language Disorders.

Sumario: 1. Introducción. 2. Materiales y métodos. 2.1. Diseño y variables. 2.2. Participantes. 2.2.1.Procedimiento. 2.3. Instrumentos de evaluación. 2.4. Fase Experimental. 2.5. Materiales. 2.6. Procedimiento de análisis de datos. 3. Resultados. 3.1. Resultados de tiempo de reacción. 3.2. Resultados de tiempo de reacción por grupos. 3.3. Resultados de aciertos. 4. Discusión. 5. Conclusiones. 6. Referencias.

\footnotetext{
Esta investigación fue financiada por ANID/PIA/Fondos Basales para Centros de Excelencia FB0003.

Universidad de Concepción. Chile

maurrutia@udec.cl

$3 \quad$ Universidad de Concepción, Chile

miriam.roainostroza@gmail.com
} 
Cómo citar: Urrutia, M.; Roa Inostroza, M. (2020) Velocidad de procesamiento en la comprensión morfológica de verbos en niños preescolares con trastorno específico del lenguaje y su relación con el control inhibitorio, Revista de investigación en Logopedia 10(1), 53-66.

\section{Introducción}

El Trastorno Específico del Lenguaje (en adelante, TEL) es uno de los trastornos de la comunicación oral más frecuente en niños preescolares con prevalencias estimadas entre 6\% y 8\%, (American Psychiatric Association, 2014). En Chile se indica la presencia de TEL en el 4\% de los niños de entre 3 y 7 años (Von Keyserlink, Castro y Carrasco, 2013). Este trastorno se entiende como un anormal desarrollo de la comprensión o expresión del lenguaje y puede afectar todos o algunos de los componentes del sistema lingüístico. Se postula que los niños con TEL tienen problemas de procesamiento del lenguaje o de abstracción de la información significativa en el almacenamiento o recuperación de la memoria a corto o a largo plazo (American Speech - Language - Hearing Association, 1980).

Las primeras referencias del TEL se refieren a deficiencias en el lenguaje expresivo y a lo largo de los años se fue avanzando para descubrir los problemas específicos que presentan los niños en la comprensión y ejecución del lenguaje. Su particularidad se debe a que, a pesar de que se garantice un entorno lingüístico adecuado para el desarrollo espontáneo del lenguaje, se produce un anormal desarrollo de éste en ausencia de daño neurológico, discapacidad intelectual, trastornos de conducta o carencias ambientales (Acosta, Ramírez y Hernández, 2015). Actualmente, se discute el papel del CI no verbal en el diagnóstico de los problemas del lenguaje y se extiende la problemática a otros dominios de tipo perceptivo, motor, cognitivo, conductual o neurológico en el TEL (Mendoza, 2016). En este último punto, a nivel motor, en un estudio de niños con TEL de entre 5 a 14 años de edad con resonancia magnética funcional (fMRI) se encontró un retraso en la adquisición de hitos motores vinculado a retrasos del habla (Trauner, Wulfeck, Tallal y Hesselink, 2000). Por su parte, Narbona y Schlumberg (1999) han encontrado una asimetría volumétrica en el hemisferio derecho en los niños con TEL contrario a la población normal, donde se evidencia una asimetría hacia el lado izquierdo.

Las alteraciones del TEL se pueden dar en distintos niveles de lenguaje como léxico, morfosintáctico, fonológico y discursivo, que se manifiestan en vocabulario limitado, una alteración del componente morfosintáctico en la inflexión verbal, dificultad para pronunciar diptongos o palabras multisilábicas. A nivel gramatical, se han estudiado las dificultades de los niños con TEL en la concordancia de género y número, el procesamiento de tiempos verbales complejos, dificultad en el manejo de artículos, pronombres y clíticos (ver Hincapié, Giraldo, Castro, Lopera, Pineda y Lopera, 2007 ). En el ámbito discursivo, dificultad para comprender y expresar nociones referidas al espacio y tiempo, comprensión de metáforas, entre otros (Del Valle, Acosta y Ramírez, 2018; Soprano, 2017).

Al describir las dificultades del TEL, en términos gramaticales, los elementos morfológicos y sintácticos representan el núcleo del trastorno. La hipótesis explicativa más global es la Hipótesis Superficial o Surface Hyphotesis propuesta por Leonard (1998), que propone que los niños con TEL tienen mayor dificultad de procesamiento cuando el sistema se sobrecarga y/o la información llega muy rápido. Esto implica que los elementos menos relevantes perceptivamente son los más vulnerables y se omiten. Otros problemas relacionados con la gramática en lenguas germánicas se producen por errores frecuentes en el uso de morfemas gramaticales verbales en el pasado simple regular o pretérito perfecto simple, así también en la producción de verbos irregulares y el uso de auxiliares e inflexiones de la tercera persona singular. Tales problemas de morfología flexiva verbal no solo afectarían la producción, sino que también la comprensión, especialmente en el juicio de gramaticalidad y en el aspecto sintáctico, son frecuentes las alteraciones gramaticales como omisión de preposiciones, auxiliares y pronombres, desorganización sintáctica y sustitución de formas verbales en español. Los niños con TEL en español difieren significativamente de sus pares en la longitud de sus oraciones y en los tipos de errores no azarosos que se producen cuando aprenden las formas regulares morfosintácticas y las sobreextienden a los verbos irregulares (Aguado, 1999; Krok y Leonard, 2015; Leonard et al.; 2003, Vang Christensen y Hansson, 2012).

Las evidencias empíricas existentes en español sobre TEL se refieren a problemas lingüísticos o cognitivos vinculados a las Funciones Ejecutivas. Dentro del primer grupo, podemos destacar los hallazgos relacionados con el aspecto gramatical en que niños de 5 años con TEL muestran mayores errores de omisión y comisión en la producción de verbos, prefiriendo aquellos de tipología verbal y argumental más simple en comparación con el grupo control (Sanz, 2002). A nivel morfológico, se ha encontrado mayor dificultad para el procesamiento de flexión verbal en niños con TEL en comparación con niños con desarrollo típico de la misma edad en mediciones de habla espontánea, en términos de mayor frecuencia de errores y tipos de errores en niños con TEL (Grinstead, Lintz, Vega-Mendoza, De la Mora, Cantú-Sanchez y Flores, 2014). Por último, a nivel discursivo, los niños con TEL presentan problemas de comprensión auditiva, a nivel global, literal e inferencial al compararlos con niños con desarrollo normotípico de su misma edad, aunque no se encuentran diferencias significativas entre el grupo TEL y un grupo de su misma edad cronológica, pero igual desempeño lingüístico (Coloma, Maggiolo, Pavez, 2013).

Dentro de los problemas cognitivos, se distinguen las funciones ejecutivas. Las evidencias empíricas existentes en español como las de Quintero, Hernández, Verche, Acosta y Hernández (2013) sostienen un deterioro de los mecanismos involucrados en el aprendizaje y déficits no lingüísticos transversales al funcionamiento cognitivo como: la atención, velocidad de procesamiento o la memoria, funciones primordiales para el desarrollo del lenguaje. Las 
hipótesis que plantean Quintero et al. (2013) involucran suposiciones sobre un déficit perceptivo, déficit de la memoria de trabajo, deficiencia específica para el desarrollo lingüístico y déficit cognitivo para el procesamiento general del lenguaje. Asimismo, se sugiere que los niños con TEL tienen dificultades con habilidades de razonamiento y pensamiento de orden superior, específicamente un rendimiento significativamente más bajo que los niños típicos en tareas de funciones ejecutivas como memoria de trabajo verbal y no verbal, fluidez verbal y no verbal, inhibición no verbal, planificación no verbal y velocidad de procesamiento, incluso cuando se hicieron ajustes para sus habilidades verbales (Henry, Messer, Nash, 2012; Reichenbach, Bastian, Rohrbach, Gross, y Sarrar, 2016).

En el intento por describir las limitaciones no lingüísticas del grupo TEL, se muestran dificultades en la capacidad de atención sostenida y fracaso en la detección de estímulos. La población TEL presentaría limitaciones en su capacidad para integrar pautas sensoriales, dificultades en el procesamiento visuoespacial y lentitud de respuesta motora. En relación a la memoria no verbal, los niños con TEL presentan peores resultados que los niños con desarrollo normal del lenguaje. En cuanto a la velocidad de procesamiento de la información de tareas simples, se observan limitaciones a medida que las tareas avanzan en complejidad. Finalmente, respecto a las funciones ejecutivas, los resultados muestran que los niños con TEL aventuran hipótesis que no comprueban o actúan al azar. Además, presentan dificultades en la categorización y son más perseverantes en los errores que en sus pares. Esto último sugiere un déficit concreto en la inducción de reglas y deficiencias en la capacidad de planificación y ejecución, así como en el control inhibitorio (Buiza-Navarrete, Adrián-Torres y González-Sánchez, 2007).

Los hallazgos en habla hispana muestran que los niños con TEL presentan disfunciones ejecutivas que van más allá de las medidas verbales, lo que podría reflejar una dificultad cognitiva general, que junto con el deterioro lingüístico y narrativo, conformarían un perfil complejo del trastorno. Tales limitaciones afectarían funciones como la atención, codificación, memoria y función ejecutiva, mostrándose en esta última mayores diferencias al obtener un rendimiento inferior a sus pares en todas las variables estudiadas de las funciones ejecutivas, como son: memoria de trabajo, planificación, fluidez e inhibición y alternancia (Acosta, Ramírez y Hernández, 2015; Buiza-Navarrete et al., 2007; Quintero et al., 2013).

En relación al procesamiento de la información, existe evidencia mixta de la correlación entre la velocidad de procesamiento de los niños con TEL y habilidades del lenguaje. Los estudios han pretendido examinar la relación entre las medidas de velocidad de procesamiento y memoria de trabajo, así como la velocidad de procesamiento en diferentes tareas lingüísticas y no lingüísticas, indicando que las tareas de velocidad en niños con TEL y el hallazgo de un procesamiento lento, sería predictivo de deterioro lingüístico y presencia de un trastorno específico de lenguaje (Leonard et al., 2007, Park, Miller y Mainela-Arnold, 2015, Miller et al., 2001). En otro estudio de Schul, Stiles, Wulfeck y Townsend (2004) se propuso evaluar la velocidad y eficiencia de la orientación atencional visuoespacial, velocidad de procesamiento visual y respuesta motora, donde se observó que el grupo TEL tuvo un procesamiento visual y respuesta motora más lenta, pero con una velocidad de orientación atencional similar al grupo control. La velocidad de escaneo de la memoria a corto plazo también disminuye si la comparamos con niños con desarrollo normotípico del lenguaje, mostrando un tiempo mayor de recuperación de la memoria, que podría contribuir a los déficits lingüísticos. Lo anterior propone una tendencia de ralentización generalizada en la velocidad de procesamiento cognitivo a nivel de producción y comprensión lingüística en una gran diversidad de tareas, preferentemente en lengua inglesa (Kail, 1994; Sininger, Klatzky y Kirchner, 1989).

Las dificultades en el procesamiento verbal en lengua española son el foco principal de la presente investigación, en que se trabajó con verbos regulares e irregulares en tiempo presente y pasado para abordar la problemática fundamental de los preescolares con TEL a través de un diseño experimental original y exploratorio, que contempla la comprensión de enunciados lingüísticos a través de una tarea experimental planteada bajo el paradigma go/no go. Se utilizaron medidas de tiempo de reacción y porcentaje de errores en un paradigma go/no-go para evaluar la velocidad de procesamiento en niños preescolares con trastorno específico de lenguaje en la comprensión oral de morfología verbal, marcador característico del trastorno, y su relación con las funciones ejecutivas a través de la aplicación de una batería de pruebas neuropsicológicas. La principal hipótesis que se plantea es que los niños con TEL tendrán mayor dificultad en el procesamiento verbal que los niños con desarrollo normotípico del lenguaje en tareas de control inhibitorio, por lo que se examinará la velocidad de procesamiento en la comprensión de morfología verbal y su relación con el control inhibitorio.

La identificación de estos marcadores lingüísticos y psicológicos en su conjunto permitiría elaborar programas de intervención más eficaces que comprendan diversos dominios para la superación del TEL, como aspectos psicolingüísticos y de funciones ejecutivas. Facilitaría, además, un abordaje multidimensional e interdisciplinario, al considerar un entrenamiento intensivo en habilidades psicolingüísticas y funciones neuropsicológicas, específicamente apuntando al funcionamiento ejecutivo (Acosta et al., 2015; Quintero et al., 2013).

\section{Materiales y Método}

\subsection{Diseño y variables}

El presente estudio es un estudio cuantitativo de tipo experimental, con un diseño factorial mixto intergrupo 2 (niños con trastorno específico del lenguaje/grupo control) e intragrupo 2 (tiempo verbal: presente/pretérito perfecto simple) 
x2 (tipo de verbo: regular/irregular). Se midieron los tiempos de reacción y porcentaje de aciertos. Las covariables que se consideraron fueron Memoria de Trabajo, Inhibición Conductual y Velocidad de denominación, todas medidas con las subpruebas del Test de evaluación neuropsicológica TENI (Tenorio et al., 2012).

\subsection{Participantes}

Participaron 45 niños preescolares con diagnóstico de Trastorno Específico de Lenguaje de 5 a 6 años de edad y 46 niños pertenecientes al grupo control con desarrollo normotípico de lenguaje. Ambos grupos tienen la misma edad y cursan el segundo nivel educacional de transición de establecimientos educacionales municipalizados de la comuna de Laja, octava región de Chile. Los criterios de exclusión para la muestra fueron la presencia de déficit auditivo, lesiones cerebrales, problemas graves de conducta, sospecha de discapacidad intelectual y antecedentes de otros trastornos del desarrollo. Los niños del grupo TEL fueron diagnosticados por el profesional Fonoaudiólogo de su propio establecimiento con las pruebas formales que se exigen para este diagnóstico tales como TEPROSIF-R con índice de confiabilidad de Cronbach de 0.9, TECAL con índice de confiabilidad de 0,93 en test-retest y STSG validado con $\mathrm{t}$ Student con p $<0.001$ y un r 0.72 en la subprueba receptiva, así como un r 0.64 en la subprueba expresiva. Los niños del grupo control fueron evaluados por la investigadora responsable del estudio, también Fonoaudióloga, con el test STSG para descartar un posible trastorno específico de lenguaje no diagnosticado. Sólo se realizó la evaluación al grupo control, quienes no habían sido evaluados anteriormente, puesto que los diagnósticos TEL se realizan por derivaciones asociadas a problemas de lectura, situación que no se daba en este grupo.

\subsubsection{Procedimiento}

En primer lugar, se consiguieron las autorizaciones de los establecimientos educacionales y consentimientos informados, así como los asentimientos de los estudiantes que participaron en la muestra. A continuación, se realizó un estudio piloto que no formó parte de la muestra total del estudio. Luego de conformar los grupos de estudio, se llevó a cabo la tarea experimental y en la última fase se aplicaron las subpruebas de evaluación neuropsicológica de memoria de trabajo visual, velocidad de denominación e inhibición conductual para correlacionarlas con las variables en estudio, tales tareas se llevaron a cabo por la investigadora responsable de forma individual en las dependencias de los establecimientos participantes en una sala acondicionada para la tarea experimental.

\subsection{Instrumentos de evaluación}

Se aplicó una prueba diagnóstica (STSG) para determinar la presencia de TEL en el grupo control y subpruebas del Test de evaluación neuropsicológica (TENI) para medir las funciones ejecutivas para el análisis de los datos (Tenorio et al., 2012). A continuación, una breve descripción de las pruebas aplicadas.

Test exploratorio de la gramática española STSG: La subprueba comprensiva y expresiva consta de 23 pares de oraciones cada una con la misma categoría sintáctica evaluada. En la vertiente comprensiva se presentan un par de oraciones y el niño debe identificar las imágenes correspondientes. Por ejemplo: "El libro es de él" / "El libro es de ella"; y en la vertiente expresiva el niño debe reproducir las oraciones a partir de un ejemplo: un dibujo se llama "El niño se vistió" y el otro se llama "El niño se viste", así cuando preguntemos el nombre de las imágenes, el niño a partir del modelo debe reproducirlas con la categoría sintáctica adecuada. Ambas se miden por percentiles, considerando un rendimiento deficitario cuando es menor a percentil 10 .

Subprueba de Memoria de trabajo de TENI: Evalúa la memoria de trabajo visual, donde el participante tiene que observar y memorizar una secuencia de salida de un personaje animado por diversos agujeros, reproduciéndola en el mismo orden de presentación. La prueba aumenta de manera gradual los estímulos en una secuencia de dos a ocho elementos.

Subprueba de Inhibición Conductual de TENI: Evalúa el control inhibitorio mediante una instrucción que se le da al menor para que en un tiempo estimado de cinco minutos, no juegue con el material tecnológico (Tablet), usado en la subprueba anterior. Se mide el tiempo en segundos que se tarda en desobedecer la instrucción. Esto permite observar la inhibición conductual y regular su comportamiento según la instrucción del evaluador.

Subprueba de Velocidad de denominación de TENI: Evalúa en milisegundos la capacidad de nombrar lo más rápido posible 6 estímulos visuales conocidos por el niño repetidos en tres líneas (pelota, casa, gato, manzana, elefante, árbol). Se espera que el niño nombre todos los objetos en un tiempo determinado y se refiere a una competencia asociada al lenguaje expresivo.

\subsection{Fase Experimental}

El experimento se llevó a cabo a través de una tarea go/no go, una tarea metalingüística en la que influye el control inhibitorio y la atención, puesto que los preescolares deben inhibir las interferencias provocadas por los estímulos de la tarea, situación que reproduce lo que ocurre en la realidad cuando está leyendo. La tarea se realiza de forma individual mediante el software experimental E-Prime versión 2.0 Professional. Para ello, se presentó el estímulo go, que 
indicaba la opción de respuesta si la condición es satisfecha y el estímulo no go, que indicaba la abstención de respuesta si no se satisface la condición dada. La tarea permitió medir el tiempo de reacción de los participantes ante tipo de verbos regular e irregular y tiempo verbal presente y pretérito perfecto simple. Las respuestas obtenidas fueron la cantidad de aciertos go, cantidad de aciertos no go y el tiempo en milisegundos que empleaban los niños al responder. La tarea consistía en escuchar una oración y presionar la barra espaciadora si la oración se encontraba bien formulada. La instrucción se realizó por medio de la historia de un "marciano" que necesitaba que le enseñaran a hablar de manera correcta, así cuando el personaje hablara correctamente, el niño debía presionar la barra espaciadora y cuando no, debía inhibir su respuesta y no presionar. Todos los participantes tuvieron un periodo de prueba que se podía repetir si fuera necesario. Después aparecía un punto de fijación con una duración de $500 \mathrm{~ms}$ (pantalla 1 figura 1). Para mantener la atención de los niños, se programó un marciano animado que movía los labios cuando aparecía la frase auditiva de manera simultánea, de este modo nos asegurábamos que el niño asociara lo que escuchaba a la imagen del marciano. Las oraciones tenían como máximo una duración de 3000 ms y el tiempo de espera para la respuesta del niño fue de igualmente $3000 \mathrm{~ms}$. Las 60 oraciones de la tarea experimental se dividieron en 4 bloques de 15 oraciones cada uno para evitar la fatiga de los niños y replantear las instrucciones si es que fuera necesario. La tarea experimental en sí, descontando instrucciones y periodo de práctica duró 15 minutos aproximadamente. En la figura 1 se grafica el esquema del procedimiento experimental.

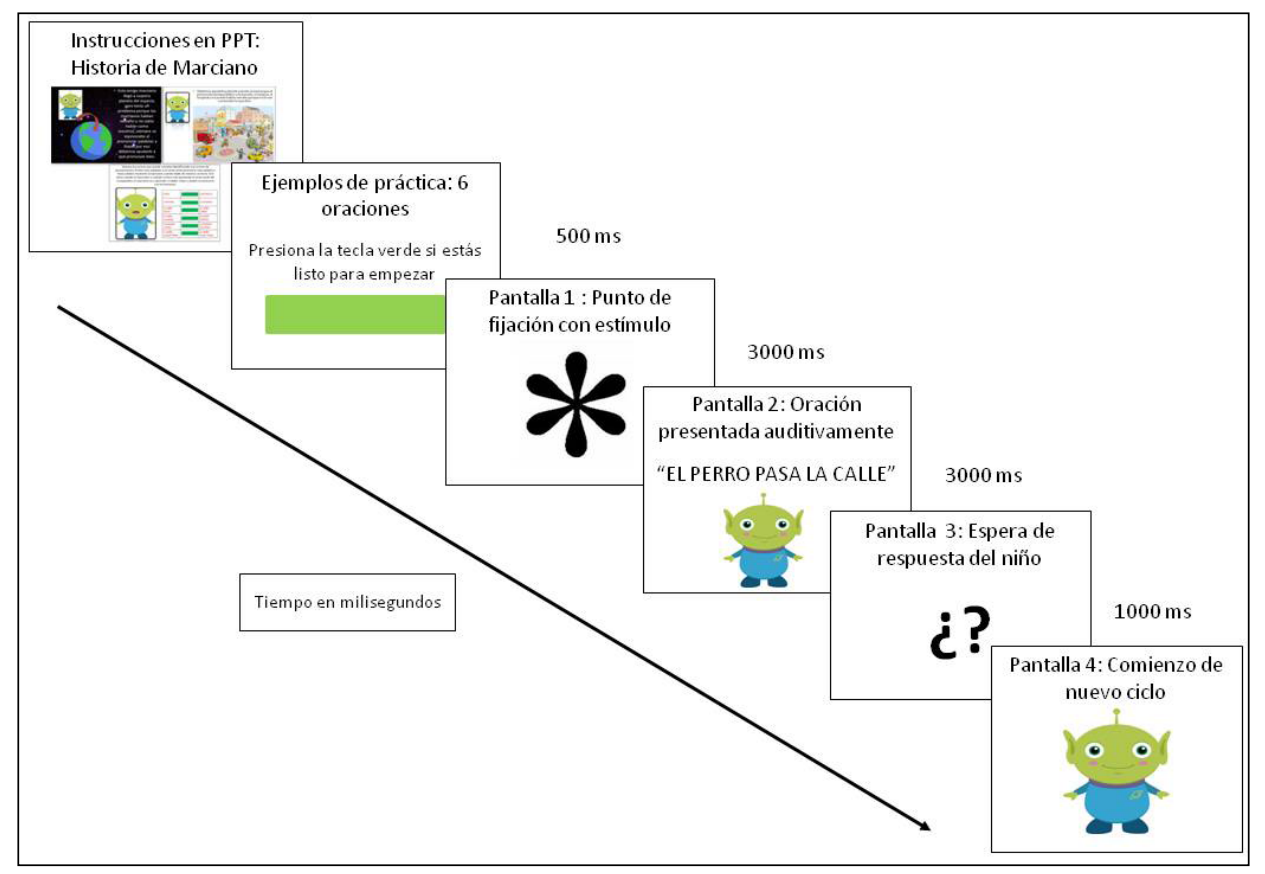

Figura 1: Esquema de tarea experimental

\subsection{Materiales}

El material lingüístico consistió en 60 oraciones de tipo sujeto-verbo-objeto por condición experimental con verbos de distinto tipo y en distinto tiempo, donde se incorporaron oraciones incorrectas ( $20 \%$ del total del material). La distribución fue la siguiente: 12 oraciones con verbos regulares en tiempo presente, 12 oraciones con verbos regulares en tiempo pretérito perfecto simple, 12 oraciones con verbos irregulares en tiempo presente, 12 oraciones con verbos irregulares en tiempo pretérito perfecto simple, 3 oraciones incorrectas con verbos regulares en tiempo presente, 3 oraciones incorrectas con verbos regulares en tiempo pretérito perfecto simple, 3 oraciones incorrectas con verbos irregulares en tiempo presente, 3 oraciones incorrectas con verbos irregulares en tiempo pretérito perfecto simple. Las estructuras bien formuladas fueron los estímulos go y las oraciones con errores fueron los estímulos no go. Se controlaron los estímulos lingüísticos por longitud y frecuencia léxica para que no hubiera diferencias significativas en estas variables entre verbos y objetos de las oraciones en estudio, esto es, oraciones con verbos regulares y oraciones con verbos irregulares. Los resultados arrojaron que no había diferencias significativas entre ambos verbos y objetos, según el análisis t student de longitud de verbos $\mathrm{t}(53)=0,843, \mathrm{p}=0,099$ y longitud de objetos $\mathrm{t}(53)=0,591$, $\mathrm{p}=0,023$; lo mismo ocurrió para el control de frecuencia léxica donde el análisis de t student arrojó lo siguiente para verbos $\mathrm{t}(53)=0,922$ y para objetos $\mathrm{t}(53)=0,890, \mathrm{p}=0,165$. El criterio usado para la formulación de las oraciones incorrectas, fue invertir las vocales de los verbos conjugados (tiró/torí; barre/berra) para crear la condición de agramaticalidad, de esta forma se controló que los niños pudieran considerarlas oraciones posibles, debido a la hiperregularización de verbos (en el anexo 1 se muestra la totalidad de estímulos usados en el experimento). Un ejemplo de las oraciones se muestra en la tabla 1. 
Tabla 1: Ejemplos de oraciones gramaticales y agramaticales

\begin{tabular}{|l|c|c|c|c|c|c|}
\hline & \multicolumn{3}{|c|}{ Tipo de oración gramatical } & \multicolumn{3}{c|}{ Tipo de oración agramatical } \\
\cline { 2 - 7 } & Sujeto & Verbo & $\begin{array}{c}\text { Complemento } \\
\text { directo }\end{array}$ & Sujeto & Verbo & $\begin{array}{c}\text { Complemento } \\
\text { directo }\end{array}$ \\
\hline $\begin{array}{l}\text { Verbo regular en } \\
\text { tiempo presente }\end{array}$ & El perro & pasa & la calle & La niña & $\begin{array}{c}\text { berra } \\
\text { (barre) }\end{array}$ & la sala \\
\hline $\begin{array}{l}\text { Verbo regular en } \\
\text { tiempo pretérito } \\
\text { perfecto simple }\end{array}$ & El bebé & pisó & la tierra & El bebé & $\begin{array}{c}\text { osú } \\
\text { (usó) }\end{array}$ & una cuchara \\
\hline $\begin{array}{l}\text { Verbo irregular en } \\
\text { tiempo presente }\end{array}$ & El hombre & pierde & la corbata & El papá & $\begin{array}{c}\text { posu } \\
\text { (puso) }\end{array}$ & la llave \\
\hline $\begin{array}{l}\text { Verbo irregular en } \\
\text { tiempo pretérito } \\
\text { perfecto simple }\end{array}$ & El niño & probó & el pastel & Yo & $\begin{array}{c}\text { midé } \\
\text { (medí) }\end{array}$ & el espejo \\
\hline
\end{tabular}

\subsection{Procedimiento de análisis de datos}

El análisis se realizó a través de un ANOVA de medidas repetidas de 2 factores, al tratarse de un diseño experimental factorial 2x2, con la incorporación de 3 covariables: la memoria de trabajo visual, velocidad de denominación e inhibición conductual. El programa estadístico fue el software SPSS, versión 22. Se realizó una limpieza de datos para identificar los outliers con un criterio estadístico que contempla la eliminación de la data de los valores que estaban \pm 2.5 por encima de la desviación estándar de la media de cada categoría lingüística. Se encontraron en la limpieza de datos 25 outliers que quedaron fuera, lo que corresponde al 10,4\% de los datos.

\section{Resultados}

\subsection{Resultados de tiempo de reacción}

Los resultados de tiempo de reacción no arrojaron efectos significativos por grupo, sin embargo, al incorporar la covariable velocidad de denominación, correspondiente a la competencia de producción lingüística en pruebas de evaluación neuropsicológica, se observan interacciones significativas por grupo, lo que permitió realizar análisis por grupos individuales. Las covariables memoria de trabajo visual e inhibición conductual no arrojaron resultados significativos.

Al incorporar la covariable velocidad de denominación se observan resultados significativos según el Tipo de Verbo $[\mathrm{F}(1,90)=4,113, \mathrm{MSE}=112351,920, \mathrm{p}=0,046]$, donde se observó un mayor tiempo de respuesta ante verbos irregulares en comparación con los verbos regulares, en ambos grupos (ver figura 2A). El verbo irregular es difícil de procesar para ambas poblaciones, indicando que es una estructura gramatical que se está adquiriendo todavía en esta etapa del desarrollo. También se encontró una interacción Verbo $\boldsymbol{x}$ Tiempo significativa $[\mathrm{F}(1,90)=3,872$, MSE $=$ $170322, \mathrm{p}=0,052]$ entre los factores, mostrándose en ambos grupos un mayor tiempo de procesamiento en el tiempo verbal pasado en comparación con el presente (ver figura 2B). La interacción se da en los tiempos de reacción, más que en la dirección de las variables, lo que implica que el tiempo presente del verbo regular es menor que la del verbo irregular presente, mientras que el tiempo pasado regular es mayor que la del tiempo pasado del verbo irregular. Una posible explicación podría estar basada en la teoría de mecanismo dual y asociación simple en el procesamiento de la inflexión verbal según Aveledo (2006), que propone que los verbos irregulares se almacenan directamente en la memoria y los verbos regulares se someten a las reglas de inflexión verbal, lo que generaría un coste cognitivo mayor.

También se encontró una triple interacción de Verbo $x$ Tiempo $x$ Grupo estadísticamente significativa, $[\mathrm{F}(1,90)=$ $4,820, \mathrm{MSE}=211990,009, \mathrm{p}=0,031]$, mostrando que los grupos se comportan de manera distinta en el tiempo de reacción según el tipo de verbo y tiempo verbal (ver figura 2C). En el análisis $t$ student, se encontraron diferencias significativas en el tiempo de reacción de verbos regulares en tiempo pasado, donde se observa un valor $\mathrm{t}(90)=3,396$, $\mathrm{p}=0,001$, que es mayor para los niños del grupo experimental en comparación con el grupo control. En cuanto a la diferencia de medias entre el tiempo de reacción de verbos regulares en tiempo presente no se observaron diferencias significativas entre los grupos con un $\mathrm{p}=0,109$, aun cuando la tendencia indica que los niños con TEL obtienen tiempos mayores. En los verbos irregulares, se encontraron diferencias significativas en las medias de verbos irregulares en tiempo presente con un valor de $\mathrm{t}(90)=2,620, \mathrm{p}=0,010$, donde nuevamente los niños con TEL obtienen tiempos de reacción mayor en comparación con el grupo control. Lo mismo ocurre en las medias de verbos irregulares en 
tiempo pasado con un valor de $\mathrm{t}(90)=2,024, \mathrm{p}=0,046$ con mayor tiempo de procesamiento en los niños TEL. Los gráficos de los resultados de tiempo con covariable velocidad de denominación se muestran en la Figura 2.

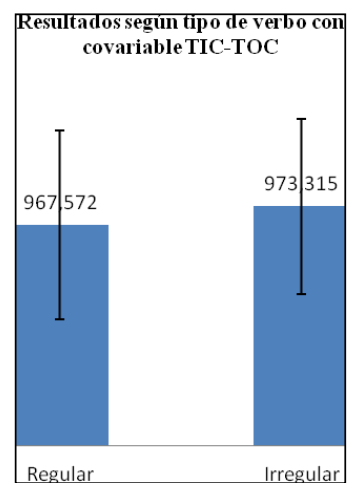

Figura 2A

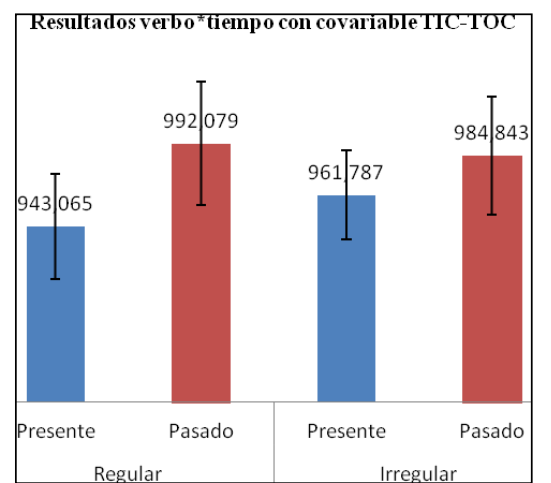

Figura 2B

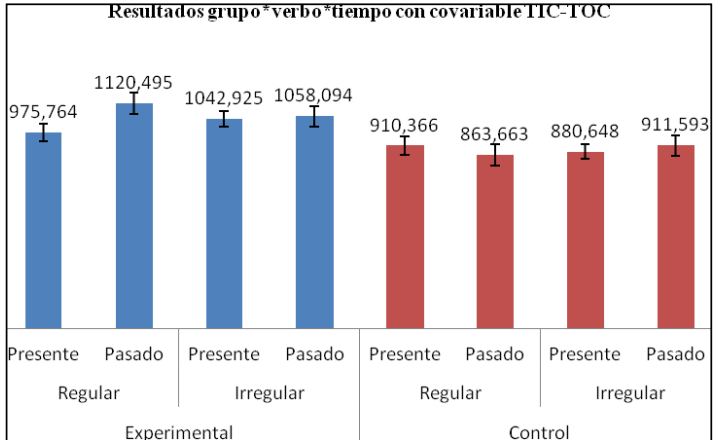

Figura 2C

Figura 2: Resultados de tiempo con covariable velocidad de denominación (TIC-TOC).

\subsection{Resultados de tiempo de reacción por grupos}

En el grupo experimental se observó un efecto significativo según Tiempo Verbal $[F(1,44)=4,197$, MSE $=$ $234049,212, p=0,046]$, donde obtuvo un mayor tiempo de reacción en el tiempo pasado en comparación con el tiempo presente (ver Figura 3A). Al incorporar la covariable velocidad de denominación se observa un efecto significativo según el Tipo de verbo $[\mathrm{F}(1,44)=4,034, \mathrm{MSE}=136789,928, \mathrm{p}=0,051]$, donde el grupo TEL obtuvo un mayor tiempo de reacción en los verbos regulares, en comparación con los irregulares (ver Figura 3B). Al incorporar la covariable memoria de trabajo, se observa un efecto significativo en la interacción de Verbo $\mathbf{x}$ Tiempo $[\mathrm{F}(1,44)=$ $3,954, \mathrm{MSE}=197411,009, \mathrm{p}=0,053]$, mostrando que los niños con TEL presentan mayor tiempo de reacción en el tiempo pasado, en comparación con el presente en ambos tipos de verbos (ver Figura 3C). Los resultados de tiempo en el grupo TEL se grafican en la Figura 3.

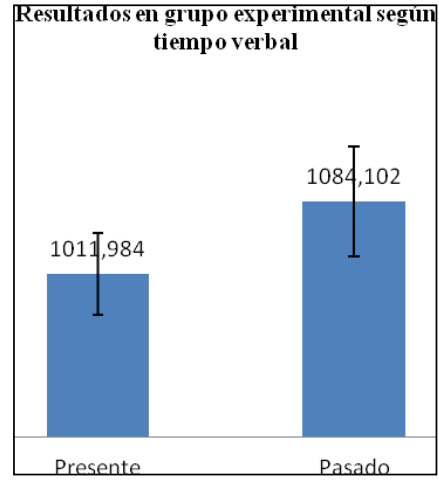

Figura 3A

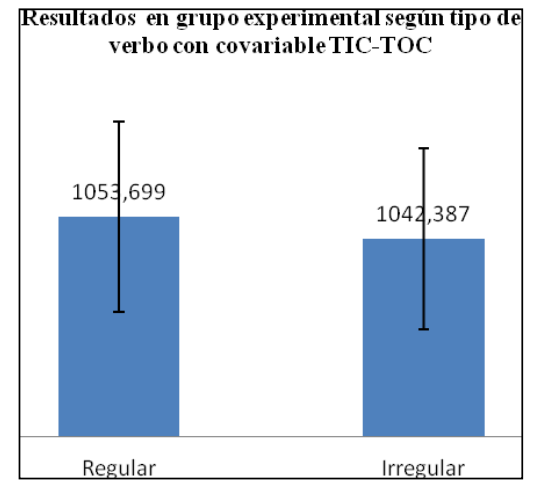

Figura 3B

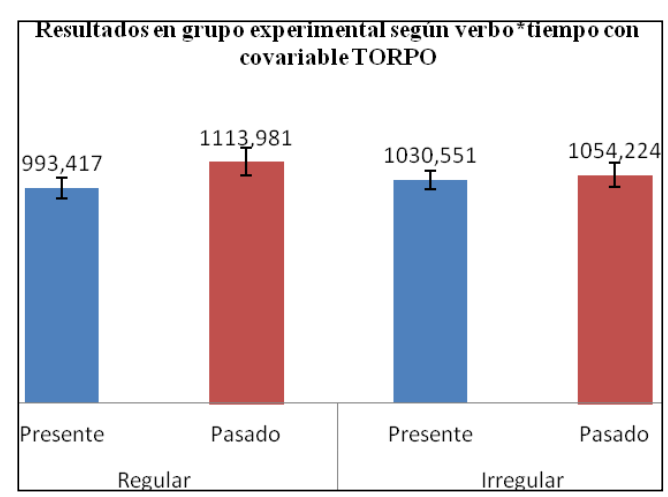

Figura 3C

Figura 3: Resultados de tiempo en grupo experimental

En el grupo control no se observaron efectos significativos en las interacciones de las variables; sin embargo, al incorporar la covariable velocidad de denominación se observan efectos según el Tiempo verbal, aquí los niños del grupo control se demoraron más en comprender el tiempo presente en relación al pasado $[\mathrm{F}(1,45)=4,479$, MSE $=181529,216, \mathrm{p}=0,040]$. Este efecto se podría explicar por la distribución temporal del evento, donde el tiempo presente podría estar actuando como evento atélico, sin punto final o término explícito. Esto influye en la velocidad de reacción llevando a un coste cognitivo mayor en relación al tiempo pasado, en que la acción fue terminada (ver Figura 4A). En la interacción Verbo $\boldsymbol{x}$ Tiempo el grupo control en el tiempo pasado se demoró más en comprender los verbos irregulares que los regulares, pero en el presente hubo mayor tiempo de reacción en el presente regular que en el presente irregular $[\mathrm{F}(1,45)=4,361, \mathrm{MSE}=153257,474, \mathrm{p}=0,043]$. La hipótesis de la distribución temporal del evento también se aplicaría en este caso, influyendo en el tiempo de procesamiento (ver Figura 4B). Los resultados de tiempo en el grupo control se grafican en la figura 4. 


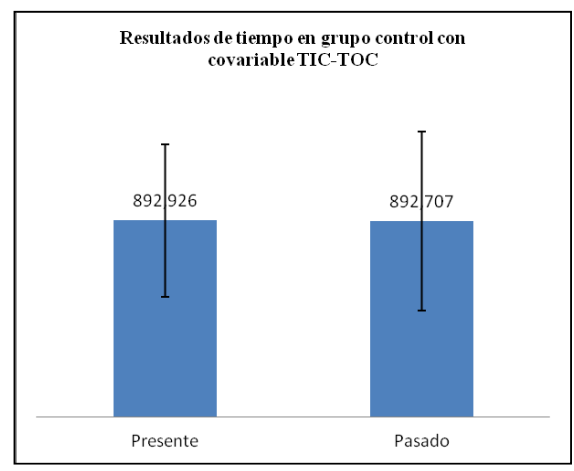

Figura 4A

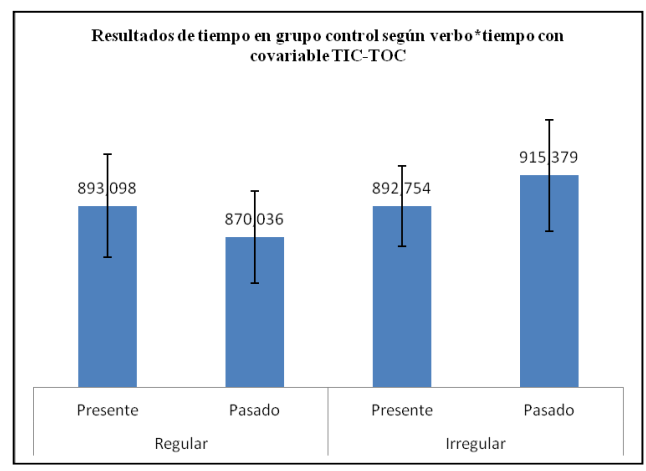

Figura 4B

Figura 4: Resultados de tiempo en grupo control con covariable velocidad de denominación

\subsection{Resultados de aciertos}

Se encontró una interacción significativa Grupo $\boldsymbol{x}$ Verbo, con una cantidad de aciertos menor en el grupo experimental, en comparación con el grupo control en el procesamiento de verbos regulares y verbos irregulares $[\mathrm{F}(1,90)=$ $5,560, \mathrm{MSE}=604,012, \mathrm{p}=0,021]$. En ambos grupos, se muestran ventajas en la cantidad de aciertos en la comprensión de verbos regulares sobre los verbos irregulares con una diferencia mayor en el grupo control (ver Figura 5A). La interacción Grupo $\boldsymbol{x}$ Tiempo también reveló efectos significativos en el grupo TEL según el tiempo verbal, con una cantidad de aciertos menor en comparación con el grupo control en ambos tiempos verbales: presente y pasado $[F(1,90)=4,877, \mathrm{MSE}=621,119, \mathrm{p}=0,03]$ (ver Figura 5B). El tiempo pasado presenta menos aciertos que el presente en el caso del grupo TEL, aunque no existan diferencias significativas entre tiempo y tipo de verbo, de acuerdo a los análisis de t student. Según estos análisis, el grupo control mostró diferencias significativas entre tiempo presente $\mathrm{y}$ pasado en los verbos irregulares $\mathrm{t}(45)=-2.554, \mathrm{p}=0.014$. Así también se encontraron diferencias significativas entre tiempo presente del verbo regular y tiempo presente del verbo irregular en el grupo control $t(45)=3.910, p=0.0001$, lo que da cuenta de que el tiempo ha modulado los resultados en relación con el tipo de verbo en este grupo. Según el Tipo de verbo se observó un efecto significativo en la cantidad de aciertos con un porcentaje menor en los verbos irregulares, en comparación con los verbos regulares en ambos grupos $[\mathrm{F}(1,90)=7,738, \mathrm{MSE}=840,581, \mathrm{p}=0,007]$ (ver Figura 5C). En la interacción Verbo $x$ Tiempo existe un efecto significativo en ambos grupos $[F(1,90)=3,841$, $\mathrm{MSE}=346,157, \mathrm{p}=0,053]$, mostrando que el porcentaje de aciertos en los dos tiempos verbales (presente y pasado) es mayor en los verbos regulares en comparación con los verbos irregulares (ver Figura 5D). En el análisis de t student se encontraron diferencias significativas en el porcentaje de aciertos en la identificación de verbos regulares en tiempo pasado, donde se observa un valor de $\mathrm{t}(90)=-3,322, \mathrm{p}=0,001$ a favor del grupo control con un mayor porcentaje de aciertos en comparación con el grupo TEL. También se encontraron diferencias significativas en las medias de verbos irregulares en tiempo pasado con un valor de $\mathrm{t}(90)=-3,215, \mathrm{p}=0,002$ donde igual se muestran resultados en la cantidad de aciertos a favor del grupo control. A diferencia del verbo regular en tiempo presente y verbo irregular en tiempo presente con un $\mathrm{p}=0,005$ y $\mathrm{p}=0,674$ respectivamente, donde no se encontraron diferencias significativas entre el porcentaje de aciertos en ambos grupos. Los resultados generales de aciertos se grafican en la figura 5 .

En la interacción Verbo $\boldsymbol{x}$ Tiempo se obtuvo una mayor cantidad de aciertos en los verbos regulares que en los irregulares en ambos tiempos verbales (presente y pasado), sin embargo si comparamos entre tiempos verbales, nuevamente el tiempo presente de los verbos irregulares obtuvo un peor desempeño en comparación con los verbos regulares, contrario a la lógica de que el tiempo presente tendría siempre mayor cantidad de aciertos $[\mathrm{F}(1,90)=6,048$, $\mathrm{MSE}=515,859, \mathrm{p}=0,016]$. Este fenómeno que se repite con la covariable velocidad de denominación y en los resultados de tiempo, se puede explicar de acuerdo a la telicidad del verbo, puesto que los verbos en tiempo presente que demuestran acciones que aún se están realizando requieren una mayor carga cognitiva, en comparación con el tiempo pasado que muestra acciones ya terminadas. Esto implica una disminución en los tiempos de reacción y, por consiguiente, en el porcentaje de aciertos.

Al incorporar las covariables de velocidad de denominación y memoria de trabajo se mantienen las interacciones halladas en los resultados generales, por lo que podemos concluir que estas variables no tienen un efecto modulador en las interacciones Grupo x Verbo y Grupo x Tiempo, sino que los resultados se deben a las características de la muestra y no a las covariables. Aún así, en el caso de la memoria de trabajo se encontró una triple interacción Verbo $\mathbf{x}$ Tiempo $\mathbf{x}$ Memoria de Trabajo Visual $[\mathrm{F}(1,90)=5.586$, MSE $=788.206, \mathrm{p}=0.017]$ graficada en la figura 6, lo que indica que la variable memoria de trabajo influye en los resultados en ambos grupos, aun cuando se conserve la interacción Verbo x Tiempo hallada en los resultados generales . De todas las funciones ejecutivas, la memoria de trabajo visual es la única que interactúa con las demás variables del diseño, por lo que se podría deducir que es la más importante de todas. 


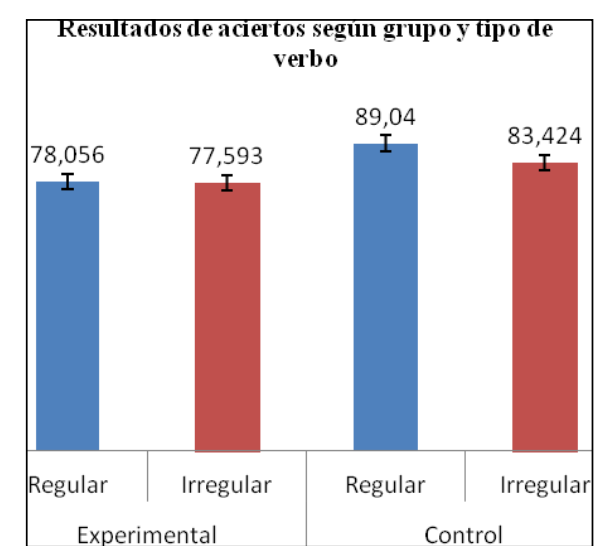

Figura 5A

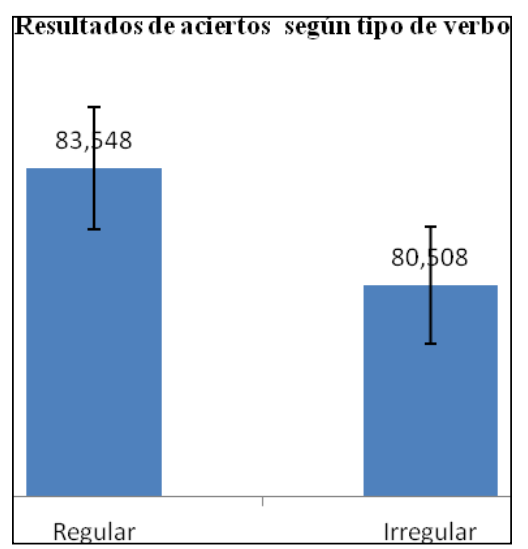

Figura 5C

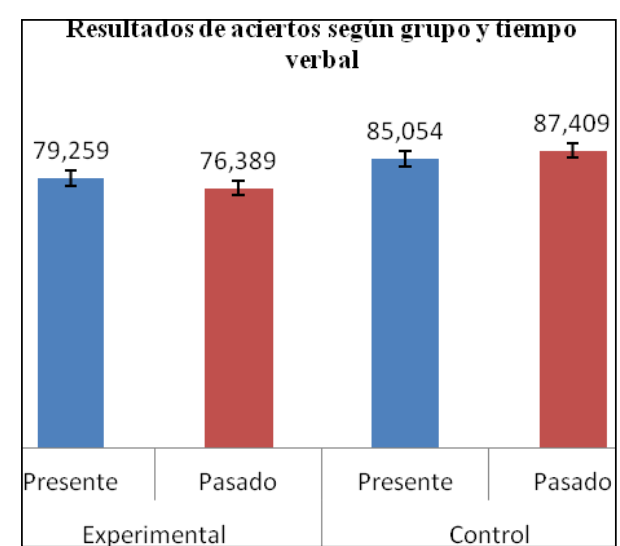

Figura 5B

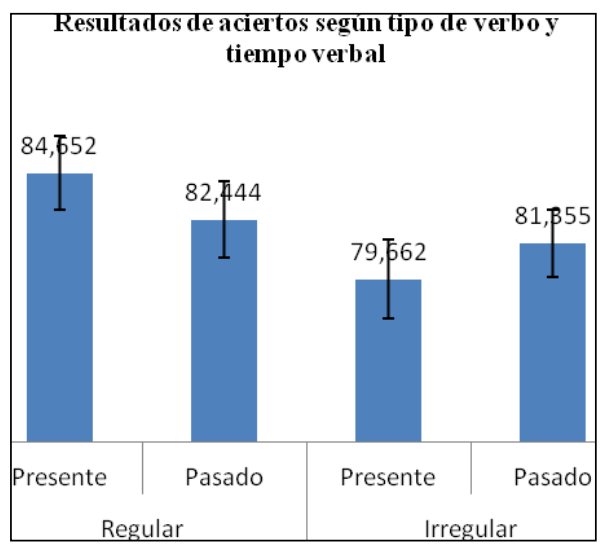

Figura 5D

Figura 5: Resultados de aciertos según grupo

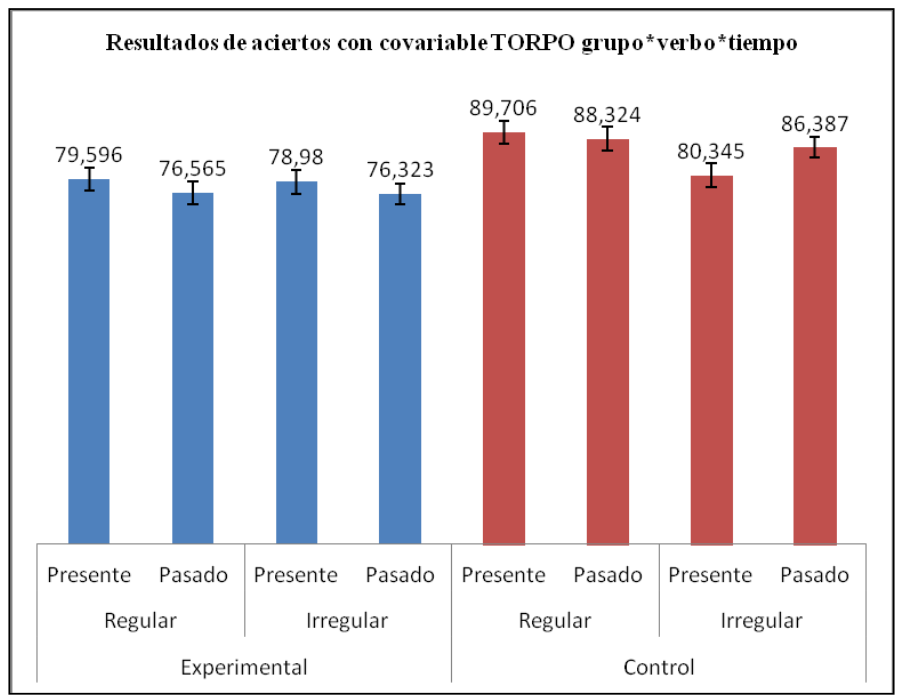

Figura 6: Resultados de aciertos con covariable memoria de trabajo 


\section{Discusión}

La exploración en la comprensión morfológica de verbos relacionada con el comportamiento ejecutivo de niños con trastorno específico de lenguaje tiene relevancia, porque a pesar de la gran heterogeneidad que se describe en el diagnóstico, todos manifiestan dificultades gramaticales. La complejidad morfológica en cuanto al tipo de verbo y tiempo verbal, mostraron en ambos grupos mayor tiempo de reacción en la comprensión de verbos irregulares y verbos en tiempo pretérito perfecto simple, y al incorporar la variable de grupo, el grupo experimental siempre obtuvo mayor tiempo de reacción en la comprensión de verbos de distinto tipo y en distinto tiempo. El tiempo de reacción en el grupo TEL fue mayor en la comprensión de verbos en tiempo pasado en comparación con el presente, postulando que las dificultades en este tipo de variables, no solamente se encuentran en la vertiente expresiva o en el uso de estos morfemas gramaticales, sino que también afectan la comprensión morfológica de estos aspectos, lo que se relaciona con lo planteado por Krok y Leonard (2015), Leonard et al.(2003) y Vang Christensen y Hansson (2012), quienes a través de estudios de metaanálisis y de tipo experimental revelaron que los niños con TEL tienen más dificultades para producir y marcar los verbos en tiempo pasado que sus compañeros con desarrollo normal del lenguaje. Tales resultados apoyan la idea de que las dificultades en el aspecto morfológico del tiempo pasado es un marcador clínico potencial en los niños con TEL, que puede darse en otras lenguas como el español y no solo en el inglés.

Los resultados actuales muestran que la velocidad de denominación, una variable asociada a la producción lingüística, influye como una covariable en los tiempos de reacción en la comprensión de oraciones con verbos regulares e irregulares, mostrando que el grupo experimental se demora más tiempo en procesar los verbos regulares en comparación con los verbos irregulares. Tal resultado se podría justificar con la teoría de mecanismo dual y asociación simple de Aveledo (2006), que propone que los verbos irregulares se almacenan en la memoria como palabras completas y no están afectos en edades tempranas a la aplicación de reglas de inflexión como ocurre en los verbos regulares que son altamente predecibles y dependen de factores como la frecuencia. En este sentido, los verbos irregulares, al ser menos frecuentes y almacenarse directamente en la memoria representarían una menor carga cognitiva en la tarea de procesamiento porque no pasarían por el proceso de análisis morfológico para aplicar las reglas de inflexión verbal propias de la lengua como ocurre en los verbos regulares, sino que se accederían por una ruta directa. Esta explicación podría justificar el mayor tiempo de reacción que tardan los niños con TEL en el procesamiento de verbos regulares. En este sentido, la memoria tendría un efecto significativo en el procesamiento de la morfología verbal, ya que según la teoría de Aveledo (2006) los sistemas de aprendizaje de verbos se llevan a cabo por rutas diferentes. Se podría suponer que en edades tempranas, donde la memoria se encuentra aún en desarrollo, falla la identificación de verbos irregulares, generando la hiperregularización, de acuerdo a la similitud y la frecuencia del verbo. Esto implica que a mayor frecuencia los verbos irregulares están más reforzados en la memoria, lo que conlleva a menos hiperregularizaciones. Por su parte, los verbos regulares no dependen de la frecuencia de uso porque no son memorizados, sino que se procesan a través de reglas en el módulo morfológico (Mendoza, Fresneda, Muñoz, Carballo y Cruz, 2001).

Al observar la relación de la memoria de trabajo visual con los tiempos de reacción, podemos decir que esta influye en la interacción Verbo x Tiempo, puesto que el grupo TEL presenta un mayor tiempo de reacción en la comprensión de verbos en tiempo pasado que en presente. Por otro lado, al observar la relación de la morfología verbal con el carácter inhibitorio de la tarea experimental, los resultados mostraron que el grupo TEL obtuvo un menor porcentaje de aciertos en ambos tipos de verbos y ambos tiempos verbales, pasado y presente, en comparación con el grupo control. Ahora bien, si comparamos sólo el tiempo verbal en el grupo TEL, éste obtuvo un menor porcentaje de aciertos en la comprensión de verbos en tiempo pasado. Tales resultados indican que existe relación entre el control inhibitorio de la tarea experimental planteada y la morfología verbal, donde la complejidad de la información lingüística no influye sólo en los tiempos de reacción, sino que también en la capacidad de inhibir respuestas automáticas o controlarlas ante una condición dada, específicamente ante tareas de morfología compleja, como en la comprensión de verbos irregulares y verbos en tiempo pretérito perfecto simple. Este efecto es interesante porque los mecanismos de control inhibitorio se inician a los 4 años edad y alcanzan su máximo desempeño en la niñez tardía (Flores-Lázaro et al., 2014, Musso, 2009), y a pesar de que en ambos grupos esta capacidad se encuentra en desarrollo, igual se observan diferencias significativas entre los grupos, mostrando una mayor dificultad en el grupo TEL. Esto último tienen relación con lo que plantean Buiza-Navarrete et al., (2007), Acosta et al., (2015) y Quintero et al., (2013) sobre las disfunciones ejecutivas que tienen los niños con TEL. Al comparar el tipo de verbo en ambos grupos, se observó un mayor porcentaje de aciertos en la comprensión de verbos regulares en comparación con los verbos irregulares. De acuerdo a la edad de los participantes y al proceso de aprendizaje de verbos y su frecuencia, se obtienen mejores resultados en la comprensión de verbos regulares que son más frecuentes y constituyen la inmensa mayoría del repertorio verbal.

Un resultado inesperado se observa en la interacción verbo $\mathbf{x}$ tiempo y grupo $\mathbf{x}$ tiempo donde se obtuvo un porcentaje mayor de aciertos en la comprensión de verbos irregulares en tiempo pasado en comparación con verbos en tiempo presente, solo en el grupo control. Este efecto se podría explicar según lo que plantea Leonard (2015) sobre la distribución temporal del evento, planteando que existe una distinción entre los eventos con punto final y sin punto final, evento télico para el primero y atélico para el segundo. En este caso, el tiempo presente actuaría como un evento atélico, sin término explícito, influyendo en el tiempo de procesamiento, lo que significaría un coste cognitivo mayor 
en relación al tiempo pasado, en donde la acción ya fue terminada, esto se relaciona con la cantidad de aciertos en este tipo de verbos, mostrando mejores resultados en los verbos en tiempo pasado por el menor coste cognitivo que conlleva la tarea de procesamiento.

La contribución más importante de este estudio plantea que el tipo de verbo y tiempo verbal influyen en los tiempos de reacción a nivel de comprensión verbal de niños con trastorno específico de lenguaje, presentando un procesamiento más lento en la comprensión de verbos irregulares y verbos en tiempo pasado, lo que se relaciona con el control inhibitorio, ya que según el porcentaje de aciertos en la tarea go/ no go, también presentaron dificultades para inhibir respuestas automáticas en ambos tipos de verbos y ambos tiempos verbales en comparación con el grupo control.

En cuanto a los nuevos hallazgos encontrados en relación a las hipótesis de procesamiento dual de verbos y comprensión de eventos télicos, se sugiere que a futuro nuevas investigaciones pudiesen estudiar en detalle estos temas en niños con trastorno específico de lenguaje y consideren las clasificaciones de este diagnóstico, debido a su gran heterogeneidad, aspecto que no pudo abordarse a cabalidad en el presente estudio.

\section{Conclusiones}

Los niños con trastorno específico de lenguaje se demoran más tiempo en la identificación de verbos irregulares que en los verbos regulares en comparación con el grupo control. Asimismo, se demoran más tiempo en la identificación de verbos en tiempo pretérito perfecto simple en comparación con el grupo control.

Los niños con trastorno específico de lenguaje obtienen una mayor tasa de errores en la tarea experimental go/ no-go y mayor tasa de errores en la comprensión de ambos tipos de verbos, regulares e irregulares y ambos tiempos verbales, pretérito perfecto simple y presente, en comparación con el grupo control. El paradigma experimental utilizado es similar a las situaciones reales de comprensión verbal a las que está expuesto un niño, en las que siempre hay factores distractores que ponen a prueba su capacidad de control inhibitorio, pues en este tipo de trastornos como el TEL, factores externos ejercen una interferencia sobre los procesos cognitivos automatizados de lectura.

La covariable velocidad de denominación, correspondiente a la producción del habla, modula los tiempos de reacción en la comprensión de verbos regulares e irregulares en ambos grupos de estudio. La memoria de trabajo visual interactuó solo con el grupo experimental en los tiempos de reacción e interactuó en los aciertos de ambos grupos de estudio, aunque no modula los resultados. Este hecho es relevante, pues hasta entonces la mayor parte de la evidencia sobre memoria y TEL está relacionada con la memoria verbal, no visual como en este caso. Las covariables velocidad de denominación e inhibición conductual interactúan con los resultados de aciertos en ambos grupos estudiados sin modularlos, por lo que los resultados se deben a las características propias de la muestra y no de la acción de éstas sobre los resultados.

La identificación de los marcadores lingüísticos y psicológicos en su conjunto permitiría elaborar programas de intervención más eficaces que comprendan diversos dominios para la superación del TEL en un abordaje multidimensional e interdisciplinario.

\section{Referencias}

Acosta, V., Ramírez, G. M. y Hernández, S. (2015). Funciones ejecutivas y lenguaje en subtipos de niños con Trastorno Específico del Lenguaje. Neurología. Extraído de http://dx.doi.org/10.1016/j.nrl.2015.12.018.

Aguado, G. (1999). Trastorno Especifico del Lenguaje. Retraso de Lenguaje y Disfasia. Málaga, España: Ediciones Aljibe.

American Psychiatric Association (APA). (2014). Manual Diagnóstico y Estadístico de los Trastornos Mentales DSM-V. Barcelona: Masson.

ASHA. (1980). Committee on Language, Speech and Hearing Services in Schools. Definitions for communicative disorders and differences. ASHA, 22, 317-318.

Aveledo, F. (2006). El procesamiento de verbos regulares e irregulares en el español infantil: mecanismo dual vs. Conexionismo. Boletín de Lingüística, 18(26), 5-32.

Buiza-Navarrete, J.J., Adrián-Torres, J.A. y González-Sánchez, M. (2007). Marcadores neurocognitivos en el trastorno específico del lenguaje. Revista de Neurología, 44, 326-33.

Coloma, C., Maggiolo, M. Pavez, M. (2013). Comprensión de narraciones orales en niños con Trastornos Específicos del Lenguaje. Actualidades en Psicología, 27(115), 129-140.

Del Valle, G., Acosta, V. y Ramírez, G. (2018). La producción gramatical en el discurso narrativo del alumnado con Trastorno Específico del Lenguaje (TEL). Signos, 51(98), 264-284.

Flores-Lázaro, J.C., Castillo-Preciado, R.E. y Jiménez-Miramonte, N.A. (2014). Desarrollo de funciones ejecutivas, de la niñez a la juventud. Anales de Psicología, 30, 463-473.

Grinstead, J., Lintz, P., Vega-Mendoza, M., De la Mora, J., Cantú-Sanchez, M. y Flores, B. (2014): Evidence of optional infinitive verbs in the spontaneous speech of Spanish-speaking children with SLI. Lingua 140, 52-66. 
Henry, L.A., Messer, D.J., Nash G., (2012). Executive functioning in children with specific language impairment. Journal of Child Psychology and Psychiatry and Allied Disciplines, 53, 37-45.

Hincapié, L., Giraldo, M., Castro, R. Lopera, F., Pineda, P. y Lopera, E. (2007). Propiedades lingüísticas de los trastornos específicos del desarrollo del lenguaje. Revista Latinoamericana de Psicología, 39(1), 47-61.

Kail, R. (1994). A method of studying the generalized slowing hypothesis in children with specific language impairment. Journal of Speech and Hearing Research, 37, 418-421.

Krok, W. C y Leonard, L. B. (2015). Past Tense Production in Children With and Without Specific Language Impairment Across Germanic Languages: A Meta-Analysis. Journal of Speech Language and Hearing Research, 58(4), 1326-40.

Leonard, L. (1998). Children with specific language impairment. MIT Press, Cambridge, MA.

Leonard, L. (2015). Time-related gramatical use by children with SLI across language: beyond tense. International Journal of Speech-Language Pathology, 17(6), 545-555.

Leonard, L. B., Deevy, P., Miller, C. A., Rauf, L., Charest, M. y Kurtz, R. (2003). Surface forms and grammatical functions past tense and passive participle use by children with Specific Language Impairment. Journal of Speech, Language, and Hearing Research, 46, 43-55.

Leonard, L., Weismer, S., Miller, C., Francis, D., Tomblin, J. B. y Kail, R. (2007). Speed of Processing, Working Memory, and Language Impairment in Children. Journal of Speech, Language, and Hearing Research, 50, 408-428.

Mendoza, E., Fresneda, M.D., Muñoz, J., Carballo, G. y Cruz, A. (2001). Morfología verbal: Estudio de las irregularizaciones de pseudoverbos en niños españoles, Psicológica 22, 165-190.

Mendoza, E. (2016). Trastorno específico del lenguaje (TEL). Avances en el estudio de un trastorno invisible. Madrid: Editorial Pirámide.

Miller, C., Kail, R., Leonard, L., y Tomblin, B. (2001).Speed of processing in children with Specific Language Impairment. Journal of Speech, Language, and Hearing Research, 44, 416-433.

Musso, M. (2009). Evaluación de funciones ejecutivas en niños: análisis y adpatación de pruebas en un contexto escolar. Revista Iberoamericana de Diagnóstico y Evaluación Psicológica, 27(1), 157-178.

Narbona, J. y Schlumberg, E. (1999). Trastornos específicos del desarrollo del lenguaje: bases neurobiológicas. Revista de Neurología, 28(2), 105-109.

Pavez, M.M. (2005). Test Exploratorio de Gramática Española de A. Toronto. $2^{\circ}$ edición. Santiago: Ediciones Universidad Católica de Chile.

Park, J., Miller, C. y Mainela-Arnold, E. (2015). Processing Speed Measures as Clinical Markers for Children with Language Impairment. Journal of Speech, Language, 58, 954-960.

Quintero, I., Hernández, S., Verche, E., Acosta, V y Hernández, A. (2013). Disfunción ejecutiva en el Trastorno Específico del Lenguaje. Revista de Logopedia, Foniatría y Audiología, 33, 172-178.

Reichenbach, K., Bastian, L., Rohrbach, S., Gross, M. y Sarrar, L. (2016). Cognitive functions in preschool children with specific language impairment. Otorhinolaryngology, 86, 22-26.

Sanz, M. (2002). Los verbos en niños con Trastorno del Lenguaje. Revista de Logopedia Foniatría y Audiología, 22(2), $100-110$.

Schul, R., Stiles, J., Wulfeck, B. y Townsend, J. (2004). How 'generalized' is the 'slowed processing' in SLI? The case of visuospatial attentional. Neuropsicología, 42(5), 661-671.

Sininger, Y. S., Klatzky, R. L. y Kirchner, D. M. (1989). Memory scanning speed in language-impaired children. Journal of Speech \& Hearing Research, 32(2), 289-297.

Soprano, A. M. (2017). “Lenguaje”. En Natalio Fejerman y Nora Grañana (comps.). Neuropsicología Infantil (pp.289-322). Buenos Aires: Paidós.

Tenorio, M., Arango, P., Aparicio, A., Benavente, C., Thibaut., C. y Rosas, R. (2012). Test de Evaluación Neuropsicológica Infantil Teni. Santiago de Chile: CedeTi UC.

Trauner, D., Wulfeck, B., Tallal, P. y Hesselink, J. (2000). Neurologic and MRI profiles of children with developmental language impairment. Developmental Medicine and Child Neurology, 42, 470-475

Vang Christensen, R. y Hansson, K. (2012). The use and productivity of past tense morphology in specific language impairment: an examination of Danish. Journal of Speech Language and Hearing Research, 55(6):1671-89.

Von Keyserlingk, L., Castro, P. J. y Carrasco, J. (2013). Teorías subjetivas de profesionales de escuelas de lenguaje en Chile sobre el trastorno específico del lenguaje. Revista CEFAC, 15(4), 873-883.

\section{Agradecimientos}

Agradecemos a Víctor M. Romeo Jiménez del S.A.I. (Servicio de Apoyo a la Investigación) de la Universidad de La Laguna por la programación del experimento en el software E-Prime y al profesor Hernán Pérez Muñoz de la Facultad de Humanidades y Arte por facilitar las dependencias del Laboratorio de Fonética de la Universidad de Concepción y Francisco Nocetti Anziani, Ingeniero en Sonido que ayudó con la grabación y edición del material lingüístico requerido 
Anexo 1. Material Lingüístico: Oraciones de verbos irregulares

\begin{tabular}{|c|c|c|c|c|c|}
\hline \multicolumn{3}{|c|}{ Verbo Irregular Tiempo Presente } & \multicolumn{3}{|c|}{ Verbo Irregular Tiempo Pasado } \\
\hline Sujeto & Verbo & Objeto & Sujeto & Verbo & Objeto \\
\hline El niño & Sabe & los colores & El niño & supo & los colores \\
\hline El perro & Sigue & al camión & El perro & siguió & al camión \\
\hline El bebé & Rueda & una pelota & El bebé & rodó & una pelota \\
\hline El hombre & Pierde & la corbata & El hombre & perdió & la corbata \\
\hline Yo & merezco & un regalo & Yo & merecí & un regalo \\
\hline Los niños & Sienten & la radio & Los niños & sintieron & la radio \\
\hline La mujer & Quiere & un café & La mujer & quiso & un café \\
\hline La señora & Tiende & la ropa & La señora & tendió & la ropa \\
\hline El hombre & Aprieta & el botón & El hombre & apretó & el botón \\
\hline La mamá & Hierve & el agua & La mamá & hirvió & el agua \\
\hline El papá & Mueve & la mesa & El papá & movió & la mesa \\
\hline El niño & Muestra & la cara & El niño & mostró & la cara \\
\hline El tío & Devuelve & la moto & El tío & devolvió & la moto \\
\hline El niño & Prueba & el pastel & El niño & probó & el pastel \\
\hline El hombre & Trae & el reloj & El hombre & trajo & el reloj \\
\hline El niño & vuela & la cometa & El niño & voló & la cometa \\
\hline El hombre & Cuelga & el cuadro & El hombre & colgó & el cuadro \\
\hline La mamá & Fríe & las papas & La mamá & frió & las papas \\
\hline El bebé & Quiebra & el huevo & El bebé & quebró & el huevo \\
\hline Yo & apuesto & unas fichas & Yo & aposté & unas fichas \\
\hline Yo & Empiezo & el juego & Yo & empecé & el juego \\
\hline El papá & Almuerza & arroz & El papá & almorzó & arroz \\
\hline La mujer & Suelta & los pájaros & La mujer & soltó & los pájaros \\
\hline El hombre & Fuerza & la cadena & El hombre & forzó & la cadena \\
\hline Ellas & Repiten & las vocales & Ellas & repitieron & las vocales \\
\hline Nosotros & Damos & la partida & Nosotros & dimos & la partida \\
\hline La maestra & Cuenta & un cuento & La maestra & contó & un cuento \\
\hline La mujer & Elige & los zapatos & La mujer & eligió & los zapatos \\
\hline Ellos & Oyen & su música & Ellos & oyeron & su música \\
\hline Ellas & visten & sus muñecas & Ellas & vistieron & sus muñecas \\
\hline Los niños & Juegan & fútbol & Los niños & jugaron & fútbol \\
\hline La mamá & Siembra & los tomates & La mamá & sembró & los tomates \\
\hline La mujer & Enciende & la vela & La mujer & encendió & la vela \\
\hline La niña & Huele & las flores & La niña & olió & las flores \\
\hline Ellas & Niegan & la mentira & Ellas & negaron & la mentira \\
\hline La maestra & Suena & la campana & La maestra & sonó & la campana \\
\hline La mamá & Cuela & la comida & La mamá & coló & la comida \\
\hline Las niñas & Acuestan & al peluche & Las niñas & acostaron & al peluche \\
\hline La mamá & Calienta & la sopa & La mamá & calentó & la sopa \\
\hline El papá & Renueva & la pintura & El papá & renovó & la pintura \\
\hline La mamá & Cuece & los fideos & La mamá & coció & los fideos \\
\hline Las niñas & Tuestan & los panes & Las niñas & tostaron & los panes \\
\hline El juguete & Vale & su dinero & El juguete & valió & dinero \\
\hline El perro & Muerde & el hueso & El perro & mordió & el hueso \\
\hline
\end{tabular}




\begin{tabular}{|l|l|l|l|l|l|}
\hline \multicolumn{2}{|c|}{ Verbo Irregular Tiempo Presente } & \multicolumn{3}{c|}{ Verbo Irregular Tiempo Pasado } \\
\hline \multicolumn{1}{|c|}{ Sujeto } & \multicolumn{1}{|c|}{ Verbo } & \multicolumn{1}{c|}{ Objeto } & \multicolumn{1}{c|}{ Sujeto } & \multicolumn{1}{c|}{ Verbo } & \multicolumn{1}{c|}{ Objeto } \\
\hline Ella & Muele & las semillas & Ella & molió & las semillas \\
\hline Las niñas & Riegan & el jardín & Las niñas & regaron & el jardín \\
\hline Los niños & Conocen & el parque & Los niños & conocieron & el parque \\
\hline Ellos & Detienen & el auto & Ellos & detuvieron & el auto \\
\hline El papá & peno (pone) & la llave & El papá & posu (puso) & la llave \\
\hline La niña & lesu (luce) & su vestido & La niña & liosú (lució) & su vestido \\
\hline El papá & sarrie (cierra) & la ventana & El papá & sorré (cerró) & la ventana \\
\hline Yo & modi (mido) & el espejo & Yo & midé (medí) & el espejo \\
\hline La mamá & servi (sirve) & el postre & La mamá & siorví (sirvió) & el postre \\
\hline La niña & pedi (pide) & su jugo & La niña & piodí (pidió) & su jugo \\
\hline
\end{tabular}

